\title{
Empirical Analysis on the Characteristic Model of Girls' Sports Teaching Based on the Element Characteristics of Physiology and Psychology
}

\author{
Xinhong $\mathrm{Yan}^{1}$, Yuanyuan Fang ${ }^{2}$, Chunying $\mathrm{Cao}^{3}$ \\ ${ }^{1}$ Department of Sports, Shijiazhuang University of Economics, Shijiazhuang, China \\ yan_xinhong@126.com \\ ${ }^{2}$ Department of Sports, Shijiazhuang TIEDAO University SIFANG College, Shijiazhuang, China \\ ${ }^{3}$ Sports School, Hebei Institute of Physical Education \\ Shijiazhuang, China
}

Keywords: adaptation evaluation; feature model; practice guidance; body immune factor; environmental factors; element characteristics

\begin{abstract}
Sports education is an important part of modern education and teaching, especially the girls' sports teaching. It should cultivate the consciousness of lifelong physical exercises from the perspective of its own healthy development. And at the same time, it should lay the foundation for the healthy development of the next generation. For girls' special physiological and psychological characteristics, it constructs girls' sports teaching model which is based on the multiple characteristics analysis. Then it effectively analyses the teaching effect and students' all aspects changes, in order to provide a new efficient way to study and practice for girls sports teaching.
\end{abstract}

\section{Introduction}

With the research of girls' sports teaching increasing year by year, it reflects the positions of girls' sports teaching that is rising from the side in the sports education. Such as, XieYing develops sports teaching strategy based on the characteristics of high school girls' psychological. It aims at high school education, and pays more attention to students' personality formation and self-development. At the same time, she puts forward reasonable sports courses plan according to the psychological characteristics of high school girls. Through the comprehensive cause analysis, she seizes the disadvantages in the teaching process, so as to put forward the feasible teaching strategy. YangQi aims at girls' physiological and psychological characteristics to teach college girls' sports teaching. From the analysis of girls' education teaching practice, she emphasizes the work purpose and importance of sports teaching for teachers oneself. According to the girl's physiological and psychological characteristics, she establishes and improves scientific girl information management mechanism, and gets ideal results in practice[1]. DaiPing carries out the sports teaching according to college girls physiological, psychological characteristics. Through a lot of research, empirical analysis, it shows that the contemporary female college students exist individual difference from the physical. And it makes analysis of their emotion, such as, sensitive, implicative, low interest in sport, like static and afraid to move, poor resistance to fatigue ability and so on. Through strengthening the theoretical knowledge learning, improving the recognition of the physical education importance, inspiring girls to take an active part in sports teaching activities, and improving the teaching methods, it strengthens the female college students to make physical ability as the goal, and achieves the purpose of improving the quality of teaching. WuJinjin carries out the sports teaching based on the adolescent girls' psychological characteristics. She combines many years' practical experience with personal experience, so as to put forward reasonable teaching plan in view of the girls' psychological characteristics[2]. It sets up the girl's physical education learning confidence. At the same time, it can give play to the subjective initiative, take an active part in physical exercise, and promote the healthy development of the girl's body and mind.

\section{The physiological characteristics analysis of girls' sports teaching}

Teenager education and development play a crucial role on the journey of life. It is the most rapid physiological development time and most complex psychological change. Through the reasonable 
sports exercise and accepting scientific sports education can get the balance development of both physical and mental. In sports education curriculum practice, girls' sports education has always been the weak link of sports education teaching. Because of some influencing factors, such as teaching environment, physical and mental characteristics, the quality of teachers and so on, most girls recognition of sports education are superficial, and low interest, thus producing fear psychology for physical exercise. How to improve the girls teaching level, it should carry on the thorough analysis from the female physiological and psychological two aspects to construct feature model structure.

Figure1 shows that girls' body features include physiological structure, body shape, physical quality, three aspects. Because girls' sports course time occasionally conflict with physiological period, in that case, girls have to ask for leave. It is closely related to the characteristics of the female physiological structure[3]. During the normal menstrual cramps, pelvic cavity much bleeding. At the same time, it reduces the body immunity, and lowers blood circulation rate, so it needs special care and meticulous observation of physiological changes. There is a close contact among pelvic cavity bleeding degree, body immune coefficient and blood circulation rate factor, and it affects the female body and mind change in physiological period. As is shown in Figure 2
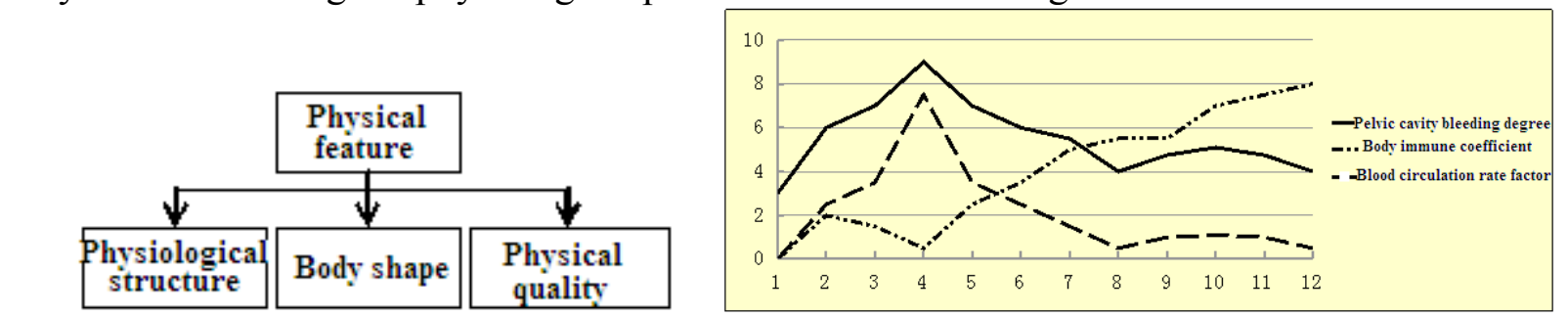

Figure 1. The model structure of girls' body feature Figure2. The relationship of important affecting factors in girls physiological period

Based on the theory of female multiple characteristics analysis, within the scope of the physiological period, the body immune coefficient can be expressed as:

$$
\vec{E}=\sum_{l=1}^{6} \vec{W}_{l}(r) \widetilde{E}_{l}
$$

$\vec{W}_{l}(r)$ is the corresponding function of the physiological initial period emotional and spiritual state, $\widetilde{E}_{l}$ is the body immune coefficient, $\vec{E}$ is the physiological initial vector line integral:

$$
\widetilde{\mathrm{E}}_{1}=\int \overrightarrow{\mathrm{E}} \cdot \mathrm{d} \overrightarrow{\mathrm{l}}=\int_{1}^{\mathrm{E}} \mathrm{E}_{1} \mathrm{dl}
$$

Vector $\vec{W}_{l}(r)$ can be expressed as two parts $\vec{W}_{l}(r)=\phi(u, v, p) \cdot \vec{q}(r), \phi(u, v, p)$ says the corresponding starting position of physical early pelvic cavity bleeding degree; $\vec{q}(r)$ says the circulation rules of the blood.

When each index meets female multiple characteristics analysis function:

Girls' physical condition enters the stable physiological stage, namely:

$$
\int \vec{W}_{i} \cdot d \vec{l}_{j}= \begin{cases}1 & i=j \\ 0 & i \neq j\end{cases}
$$

$\vec{W}_{i}$ is the psychological function. And node element in mental state function is a scalar function.

For the both sides' nodes are respectively $\mathrm{k}, \mathrm{m}$. Its mental state function can be expressed as $\mathrm{N}$ by the emotional function of node element[4]:

$$
\vec{W}_{l}=N_{k} \nabla N_{m}-N_{m} \nabla N_{k}
$$

Female multivariate analysis lays a scientific foundation for girls' sports teaching research theory, and provides practical and feasible guiding ideology.

\section{The psychological characteristics analysis of girls' sports teaching}

Due to the girls' special physiological characteristics that there are some weak places for the treatment of sports education curriculum learning. Not only the physiological characteristics affects girls' sports teaching effect, but girls' sports teaching must start from girls' psychology[5]. It should clear the interaction of psychological characteristics which constitute the girls' sports teaching bottleneck. Girls' psychological characteristics and the relationship are shown in Figure3. 
Through the analysis of female psychological characteristics associated model, it can obtain the girls' true psychology reflection on sports education curriculum which is constituted by each two factors. Accordingly, it makes targeted questionnaires, and conducts a sample survey on the school girls. Based on the analysis of survey results, it sums up the affecting results of students' psychological characteristics, which are obtained by the factors of teacher in the teaching practice. It is shown in Figure4.
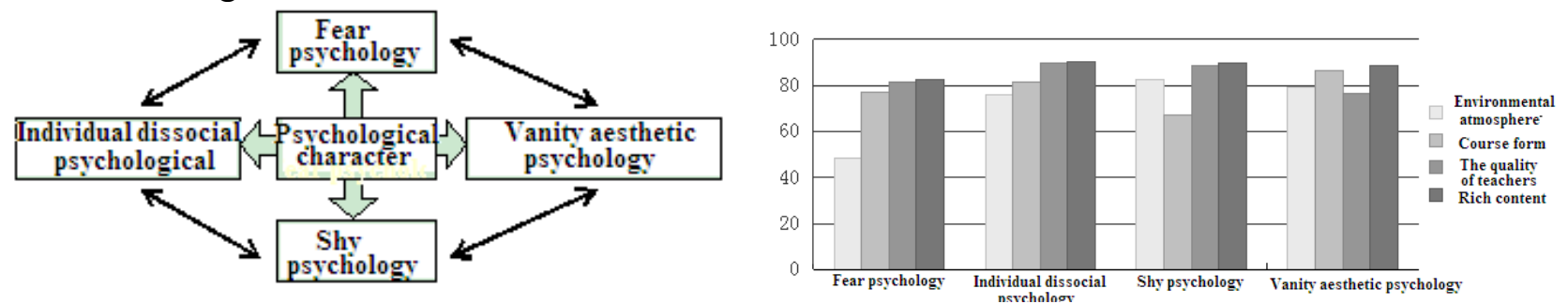

Figure3. The characteristics associated mode of girls' psychology Figure4. The influence of teaching factors on psychological characteristics

According to the above research results, it can aim at different psychological characteristics tendency students to adopt the corresponding teaching countermeasures. Then scientifically and effectively help students overcome the psychological barriers, and fundamentally improve the girls' enthusiasm of sports.

\section{Practice guidance model}

Synthesizing the important influencing factors of above girls' physiological period, girls' physical feature model structure and combining with the female psychological characteristics related model, teaching factors which are the influence of the psychological characteristics. Based on the theory of female multiple characteristics analysis creates practice guidance model of girls' education teaching.

Namely $A u=f, A=-\varepsilon \nabla^{2}$.Setting environmental factors as $\Omega$, and course content as $\left.\Gamma u\right|_{\Gamma}=f_{0}$ is for the main students who have fear psychology; $\left.\frac{\partial u}{\partial n}\right|_{\Gamma}=f_{2}$ is for the main students who have shy psychology;

$$
\begin{aligned}
& \left.\left(\frac{\partial u}{\partial n}+f_{1} u\right)\right|_{\Gamma}=f_{3} \text { is for the main students who have vanity aesthetic psychology; } \\
& \left.\left(\frac{\partial u}{\partial n}+f_{1} \frac{\partial u}{\partial n}\right)\right|_{\Gamma}=f_{4} \text { is for the main students who have dissocial psychological; }
\end{aligned}
$$

The way of solving the teaching bottleneck is trying to transform the psychological barriers into positive factors, and then according to the above formula to make repeatedly test, judge the situation of overcoming psychological barriers. Usually, it can find one favorable factor $v$ change the current teaching situation, further use the corresponding relationship between $u$ and the known factors $v[6]$ :

$$
\left\{\begin{array}{c}
u_{D}=u-v \\
A u_{D}=A u-A v \\
A u_{D}=G
\end{array}\right.
$$

It can obtain the breakthrough relationships for girls' sports teaching dilemma[7]:

$$
G=A u-A v=f-A v
$$

Knowing $\left.u_{D}\right|_{\Gamma}=0$, according to the formula 5 simultaneous the results, it can get:

$$
I\left(u_{D}\right)=<A u_{D}, u_{D}>-<u_{D}, G>-<G, u_{D}>, \delta I=0
$$

This Formula uses $u_{D}$ to represent the teaching difficult factor, also can be replaced by $u$.

$$
\begin{aligned}
I(u) & \left.=\iiint_{\Omega}-\varepsilon(u-v) \nabla^{2}(u-v) d \Omega-2 \iiint_{\Omega}\left(f+\varepsilon \nabla^{2} v\right)(u-v) d \Omega\right) \\
& =\iiint_{\Omega}\left[-\varepsilon u \nabla^{2} u-2 f u-\varepsilon u \nabla^{2} v+\varepsilon v \nabla^{2} u+2 f v+\varepsilon v \nabla^{2} v\right] d \Omega(8)
\end{aligned}
$$

But because $2 f v+\varepsilon v \nabla^{2} v$ doesn't contain $u$, the obtained corresponding conclusion can be cut out. 
Reusing the related formula which has been carried out the analysis of students' incentives according to students' age and psychological characteristics in sports teaching[8]:

$$
I(u)=-\iiint_{\Omega}\left[\varepsilon u \nabla^{2} u+2 f u\right] d \Omega-\varepsilon \iint_{\Omega}\left(u \nabla^{2} v-v \nabla^{2} u\right) d \Omega
$$

Using the excluding internal law to reduce the order number of derivative:

$$
I(u)=\iint_{\Omega} \varepsilon|\nabla u|^{2} d \Omega-\oiint_{\Gamma} \varepsilon u \frac{\partial}{\partial n} d \Gamma-\iiint_{\Omega} 2 f u d \Omega-\varepsilon \oint \oiint_{\Gamma}\left(u \frac{\partial}{\partial n}-v \frac{\partial u}{\partial x}\right) d \Gamma_{\tilde{\nu}}
$$

Eventually, it determines the absolute external influencing factors. If it gives one of the external causes, then:

$$
\frac{\varepsilon}{2} \oiint_{\Gamma}\left(u \frac{\partial v}{\partial n}-v \frac{\partial u}{\partial n}+u \frac{\partial u}{\partial n}\right) d \Gamma=\frac{\varepsilon}{2} \oiint_{\Gamma} f_{0} \frac{\partial v}{\partial n} d \Gamma
$$

Due to $\left.v\right|_{\Gamma}=\left.u\right|_{\Gamma}$, so it can eliminate two Formulas. The right Formula of equation doesn't contain $u$, so it can be cut out. Then

$$
\left\{\begin{array}{l}
I(u)=\iiint_{\Omega} \frac{\varepsilon}{2}|\nabla \varphi|^{2} d \Omega-\iiint_{\Omega} f u d \Omega=\min \\
\left.u\right|_{\Gamma}=f_{0}
\end{array}\right.
$$

In order to emphasize on the internal influence affecting degree, if it gives a kind of external conditions,

$$
\left.\frac{\partial u}{\partial n}\right|_{\Gamma}=f_{2}-\left.f_{1} u \frac{\partial v}{\partial n}\right|_{\Gamma}=f_{2}-f_{1} v
$$

When it is $f_{1}=0$, it is the absolute internal function. The strongest internal influence is[9]:

$$
\frac{\varepsilon}{2} \oiint_{\Gamma}\left(u \frac{\partial v}{\partial n}-v \frac{\partial u}{\partial n}+u \frac{\partial u}{\partial n}\right) d \Gamma=\frac{\varepsilon}{2} \oiint_{\Gamma}\left(u f_{2}-u f_{1} v-v f_{2}+v f_{1} u+u f_{2}-f_{1} u^{2}\right) d \Gamma
$$

$v f_{2}$ doesn't contain $u$, it can be cut out. Then

$$
\frac{\varepsilon}{2} \oiint_{\Gamma}\left(u \frac{\partial v}{\partial n}-v \frac{\partial u}{\partial n}+u \frac{\partial u}{\partial n}\right) d \Gamma=\frac{\varepsilon}{2} \oiint_{\Gamma}\left(2 u f_{2}-f_{1} u^{2}\right) d \Gamma
$$

Finally, it can get

$$
I(u)=\iiint_{\Omega} \frac{\varepsilon}{2}|\nabla u|^{2} d \Omega-\iiint_{\Omega} f u d \Omega-\oiint_{\Gamma}\left(\varepsilon f_{2} u-\frac{\varepsilon}{2} f_{1} u^{2}\right) d \Gamma=\min
$$

Through the above analysis from the environment factors and the teaching content of the course, it draws out the internal factors and external factors' influence screening multilayer analysis thoughts, scientifically and directly reflects the key to teaching.

In the teaching process, teacher plays a role on a guide and assist. They should adopt flexible teaching methods, and aim at all kinds of the student to carry on the patient education. Then, it needs to create a positive atmosphere for sports education teaching, keep interaction with students. Especially, for the understanding of students' psychological and counseling, teachers should inspire students' thinking in the mutual discussion, mine students' potential, and encourage students to take actively participation, so as to reach a consensus on the importance of sports education teaching.

\section{Conclusion}

With the development of the education level, sports education also presents diversified development trend. Gradually, it has thinned students' various factors, such as age, gender, physical and so on. Carrying out targeted scientific teaching mode, cultivating students to pay attention to their health and consciousness of taking part in physical exercise, so as to achieve high quality sports teaching goal, and provide reliable guarantee for the construction of citizens' health system. Through the deep investigation of girls' sports behavior and consciousness present situation in colleges and universities, it explores the regular pattern of the girls' physical exercise in college. It provides the strong basis for the reform of the sports education and improvement of the teaching process. In the framework of national education system reform and improving the health level, sports education has 
attracted lots of attention. How to solve the various complicated problems in the sports education has also become the most important thing of promoting sports teaching quality.

\section{References}

[1] Paula J Adams Hillard.Menstruation in young girls: a clinical perspective.Obstetrics \& Gynecology, 2012, 99(4): 655-662.

[2] LuHe,ZhenranGao.Participation in a summer sport-based youth development program for disadvantaged youth: Getting the parent perspective ,Children and Youth Services Review, 2012, 34(7):1367-1377.

[3] Alexis Lyras, Jon Welty Peachey,Integrating sport-for-development theory and praxis, Sport Management Review, 2011, 14(4): 311-326.

[4] Donald E. Greydanus, Dilip R. Patel,Sports Doping in the Adolescent: The Faustian Conundrum of Hors De Combat,Pediatric Clinics of North America, 2010,67(3): 729-750.

[5] Tony Xing Tan, Linda A. Camras, Social skills of adopted Chinese girls at home and in school: Parent and teacher ratings,Children and Youth Services Review, 2011,33(10): 1813-1821.

[6] Sandy K. Wurtele,Preventing the sexual exploitation of minors in youth-serving organizations, Children and Youth Services Review, 2012, 34(12): 2442-2453.

[7] Nico Schulenkorf, The roles and responsibilities of a change agent in sport event development projects,Sport Management Review, 2010,13(2): 118-128.

[8] Jari-Erik Nurmi,Students' characteristics and teacher-child relationships in instruction: A metaanalysis ,Educational Research Review, 2012, 7(3): 177-197.

[9] H. Richard Milner IV.Disrupting deficit notions of difference: Counter-narratives of teachers and community in urban education, Teaching and Teacher Education, 2008, 24(6): 1573-1598. 